\title{
Особенности формирования и развития ионизационных фронтов В предварительно ионизированной газовой среде
}

\author{
(C) В.С. Курбанисмаилов ${ }^{1}$, О.А. Омаров ${ }^{1}$, Г.Б. Рагимханов ${ }^{1, \uparrow, ~}$ \\ Д.В. Терешонок ${ }^{2}$ \\ ${ }^{1}$ Дагестанский государственный университет, Махачкала \\ ${ }^{2}$ Объединенный институт высоких температур РАН, Москва \\ 『 E-mail: gb-r@mail.ru
}

Поступило в Редакцию 2 мая 2017 г.

Исследовано влияние начальных условий на особенности формирования и развития катодонаправленной волны ионизации между двумя плоскими электродами в гелии при атмосферном давлении с применением высокоскоростного фотоэлектронного регистратора с наносекундным временны́ разрешением и на основе двумерной осесимметричной диффузионно-дрейфовой модели. Результаты численных расчетов находятся в удовлетворительном согласии с экспериментальными данными.

DOI: 10.21883/PJTF.2017.18.45036.16844

Неравновесная и нестационарная плазма импульсных объемных разрядов (OP) находит широкое применение в мощных газовых лазерах, источниках оптического излучения и т.д. Несмотря на большое число работ, посвященных исследованию импульсных объемных разрядов, многие вопросы, связанные с физикой импульсного пробоя и механизмами формирования начальных стадий, до конца не изучены и вызывают научные дискуссии [1-6].

В связи с этим несомненный интерес представляют экспериментальные и теоретические исследования последовательной динамики формирования и развития импульсного ОР в условиях предварительной ионизации газа в гелии атмосферного давления. Задача настоящей работы - определение роли неоднородной предыонизации в разрядном промежутке в формировании фронта волны ионизации. 
Экспериментальная установка и методики исследования описаны в работе [7]. Напряжение в промежутке менялось в пределах $3-20 \mathrm{kV}$. Напряжение и ток разряда регистрировались соответственно омическим делителем и малоиндуктивным шунтом с применением цифровых осциллографов типа Актаком и Tektronix. Пространственно-временное развитие разряда фиксировалось фотоэлектронным регистратором ФЭР2-1. Предварительная ионизация газа $\left(n_{0} \sim 10^{8} \mathrm{~cm}^{-3}\right)$ достигалась с помощью облучения промежутка через сетчатый анод УФ-излучением стороннего искрового разряда [8]. Исследуемый промежуток длиной в $1 \mathrm{~cm}$ был образован сетчатым анодом и сплошным катодом (диаметром $4 \mathrm{~cm})$ из нержавеющей стали.

Наибольший интерес представляют экспериментальные результаты прямых наблюдений динамики формирования разряда с пространственным и временны́м разрешением в наносекундном диапазоне времени, полученные с применением ФЭР2-1.

В частности, на рис. 1 представлены фотографии (1-4) формирования ОР в Не в различные моменты времени, полученные при наличии предварительной ионизации газа. На рисунках анод расположен сверху, а катод - снизу.

Как видно из рис. 1 (фотографии 1-4), первое регистрируемое свечение возникает на аноде после приложения внешнего поля, которое в дальнейшем в виде диффузного свечения распространяется к катоду с характерной скоростью $\sim 10^{7} \mathrm{~cm} / \mathrm{s}$. Фронт свечения неоднороден, интенсивность спадает от оси разряда к периферии, что указывает на более высокую интенсивность ионизационных процессов на оси разряда. После прихода фронта свечения на катод разряд переходит в следующую фазу - фазу объемного горения.

При малых напряжениях пробоя $U_{0}<6 \mathrm{kV}$ формируется разряд с высокой однородностью свечения и длительностью горения. При напряжениях $U_{0}>6 \mathrm{kV}$ (рис. 1 , фотография 5) и плотностях тока $j \geq 40 \mathrm{~A} / \mathrm{cm}^{2}$ в прикатодной области формируются плазменные каналы, привязанные к катодным пятнам. При увеличении числа катодных пятен появление искрового канала в промежутке задерживается, формируется множество нитевидных каналов. Установлено, что с ростом энерговклада в источник предыонизации повышаются однородность и устойчивость объемного разряда. При очень больших перенапряжениях $U_{0} \geq 12 \mathrm{kV}$ происходит зажигание разряда в режиме сильноточной диффузии [9-11].

Письма в ЖТФ, 2017, том 43, вып. 18 


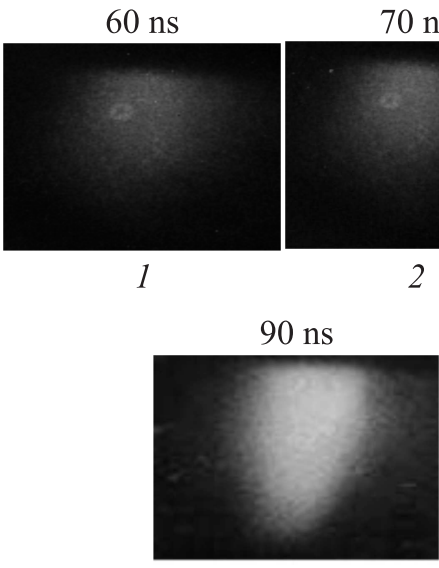

4

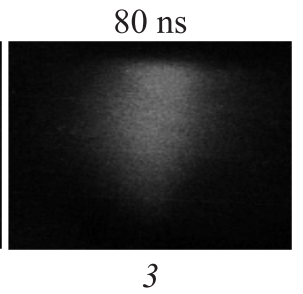

3

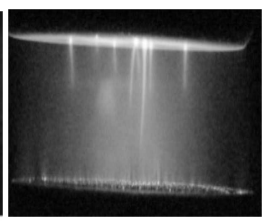

5

Рис. 1. Динамика формирования OP в Не (1-4) и интегральная картина свечения разряда (5): $p=760$ Torr, $d=1 \mathrm{~cm}, U_{0}=7 \mathrm{kV}$.

Для анализа физического механизма формирования импульсного ОР используются различные численные модели [5].

В настоящей работе для описания разряда используется двумерная осесимметричная диффузионно-дрейфовая модель движения электронов и ионов вместе с уравнением Пуассона [12-15]

$$
\begin{gathered}
\frac{\partial n_{e}}{\partial t}+\nabla \boldsymbol{\Gamma}_{e}=\alpha\left|\boldsymbol{\Gamma}_{e}\right|-\beta n_{e} n_{+}, \\
\frac{\partial n_{+}}{\partial t}+\nabla \boldsymbol{\Gamma}_{+}=\alpha\left|\boldsymbol{\Gamma}_{e}\right|-\beta n_{e} n_{+}, \\
k_{i_{-} n l}=k_{i}\left(1+\frac{\mathbf{E} D_{e} \nabla n_{e}}{\mu_{e} n_{e} E^{2}}\right), \\
\nabla \mathbf{E}=\frac{e}{\varepsilon_{0}}\left(n_{+}-n_{e}\right) .
\end{gathered}
$$

Здесь $\boldsymbol{\Gamma}_{e}=-n_{e} \mu_{e} \mathbf{E}-D_{e} \nabla n_{e}, \boldsymbol{\Gamma}_{+}=-n_{+} \mu_{+} \mathbf{E}-D_{+} \nabla n_{+}-$потоки электронов и ионов; $n_{e}$ и $n_{+}-$концентрации электронов и ионов; $\alpha-$ ионизационный коэффициент Таунсенда; $\beta-$ коэффициент

Письма в ЖТФ, 2017, том 43, вып. 18 
рекомбинации; $\mu_{e}(E / N)$ и $\mu_{+}(E / N)$ - подвижности электронов и ионов; $D_{e}=\frac{k T_{e} \mu_{e}}{e}$ и $D_{+}=\frac{k T_{+} \mu_{+}}{e}-$ коэффициенты диффузии электронов и ионов; $k_{i}$ - локальный коэффициент ионизации; $k_{i \_n l}-$ нелокальные коэффициенты ионизации $[15] ; \varepsilon_{0}-$ электрическая постоянная; $\varphi=\nabla \mathbf{E}$, где $\mathbf{E}-$ вектор напряженности электрического поля; $T_{e}(E / N)$ - температура электронов. Параметры переноса, ионизации и рекомбинации и теплофизические свойства задавались зависимостями из работ $[13,14]$. Учет нелокальности для коэффициента ионизации выполнен в соответствии с [15].

При моделировании исследовалось развитие разряда между двумя плоскими электродами с радиусом $r=2 \mathrm{~cm}$ и межэлектродным расстоянием $1 \mathrm{~cm}$, соединенными с конденсатором емкостью $C=1.5 \cdot 10^{-8} \mathrm{~F}$ через балластное сопротивление $R_{b}=2 \Omega$, которое одновременно является шунтом для определения тока разряда.

В начальный момент времени температура газа считалась равной $T_{0}=293 \mathrm{~K}$, а начальное давление исследуемого газа $-p=760$ Torr. Концентрации электронов и ионов в начальный момент времени по всей расчетной области считались неизменными и равными: $n_{e}(x, y, 0)=n_{+}(x, y, 0)=n_{0}(x-$ радиальное направление, $y$ - координата в перпендикулярном направлении от анода к катоду). Граничные условия на катоде следующие: $y=1 \mathrm{~cm}, \boldsymbol{\Gamma}_{e}=-\gamma \boldsymbol{\Gamma}_{+}, \partial n_{+} / \partial y=0$, $\varphi_{k}=0, \gamma=0.1-$ коэффициент вторичной эмиссии.

Граничные условия на аноде:

$\partial n_{e} / \partial y=0, \quad n_{+}=0, \quad \varphi_{a}=U(t)-I(t) R_{b}, \quad I_{t}=2 \pi \int_{0}^{r} j_{a}(x, t) x d x$.

Здесь $I(t)-$ общий ток в электрической цепи, $j_{a}(x, t)-$ плотность тока на аноде.

В радиальном направлении на границах расчетной области для потенциала и концентраций задавалось условие отсутствия суммарного потока частиц (равенство производных нулю). В расчете использовался явный метод с первым порядком точности по времени и пространству. Уравнение Пуассона для электрического поля рассчитывается итерационным методом. Число расчетных ячеек в радиальном направлении составляло 200, в перпендикулярном направлении - 300 (со сгущением вблизи электродов). 
Концентрации электронов и ионов предыонизации в промежутке задавались как

$$
\begin{aligned}
& n_{e}(x, y)=n_{e 0} \frac{\exp \left(-k x^{2}\right)}{r^{2}} \frac{\exp \left(-k y^{2}\right)}{d^{2}}, \\
& n_{+}(x, y)=n_{+0} \frac{\exp \left(-k x^{2}\right)}{r^{2}} \frac{\exp \left(-k y^{2}\right)}{d^{2}},
\end{aligned}
$$

где $r=2 \mathrm{~cm}-$ радиус разрядного промежутка, $d=1 \mathrm{~cm}-$ межэлектродное расстояние, $k$ - коэффициент, определяющий степень неоднородности предыонизации. Расчеты проводились для значений $k=1-10$.

Изменением температуры газа можно пренебречь, поскольку на стадии формирования разряда, как показывают расчеты, нагрев газа незначителен и не вносит изменений в физику процесса.

Рассмотрим результаты численного моделирования стадии формирования разряда в условиях неоднородной предыонизации газа. На рис. 2 приведены характерные распределения концентрации электронов и продольной составляющей поля в промежутке. Как следует из полученных результатов, после приложения высоковольтного импульса электроны вследствие дрейфа уходят из прикатодной области на анод, при этом концентрация электронов в прикатодной области уменьшается, одновременно увеличивается их концентрация в столбе за счет ионизационного размножения электронов. Появление нескомпенсированного положительного пространственного заряда усиливает поле в прикатодной области промежутка, одновременно ослабляя его в положительном столбе (рис. 2, $b$ ).

Неоднородность предыонизации газа как вдоль, так и поперек разрядного промежутка приводит к неоднородности распределения концентрации электронов поперек разрядного промежутка; следовательно, фронт волны ионизации не является плоским, а имеет вытянутую форму (рис. 2,a).

Как видно из приведенных рисунков, формирование волны ионизации преимущественно происходит на оси разрядного промежутка, где поле максимально. Скорость ионизационного фронта уменьшается от центра разрядного промежутка к периферии.

Кроме того, напряженность поля меняется не только вдоль разрядного промежутка, но и поперек. В процессе развития волны ионизации нескомпенсированный положительный заряд на фронте создает

Письма в ЖТФ, 2017, том 43, вып. 18 

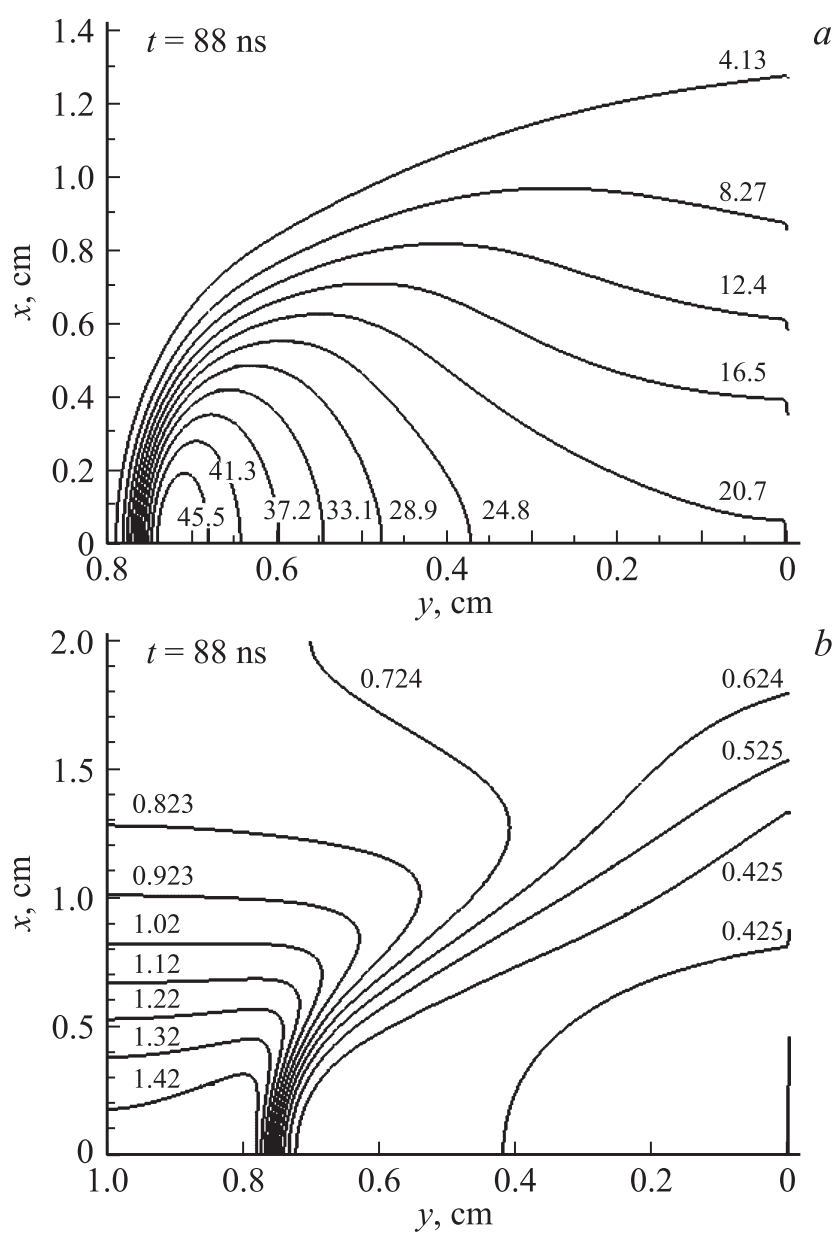

Рис. 2. Изолинии концентрации электронов $n_{e}\left(10^{10} \mathrm{~cm}^{-3}\right)(a)$ и продольной составляющей напряженности поля $E_{y}\left(10^{4} \mathrm{~V} / \mathrm{cm}\right)(b)$ в межэлектродном промежутке: $U_{0}=7 \mathrm{kV}, p=760$ Torr, $d=1 \mathrm{~cm}$.

достаточно высокое поперечное электрическое поле $E_{x}$ (рис. 3). При этом проекция $E_{x}$ на фронте волны ионизации положительна, а за фронтом имеет отрицательную составляющую. По мере приближения к

Письма в ЖТФ, 2017, том 43, вып. 18 

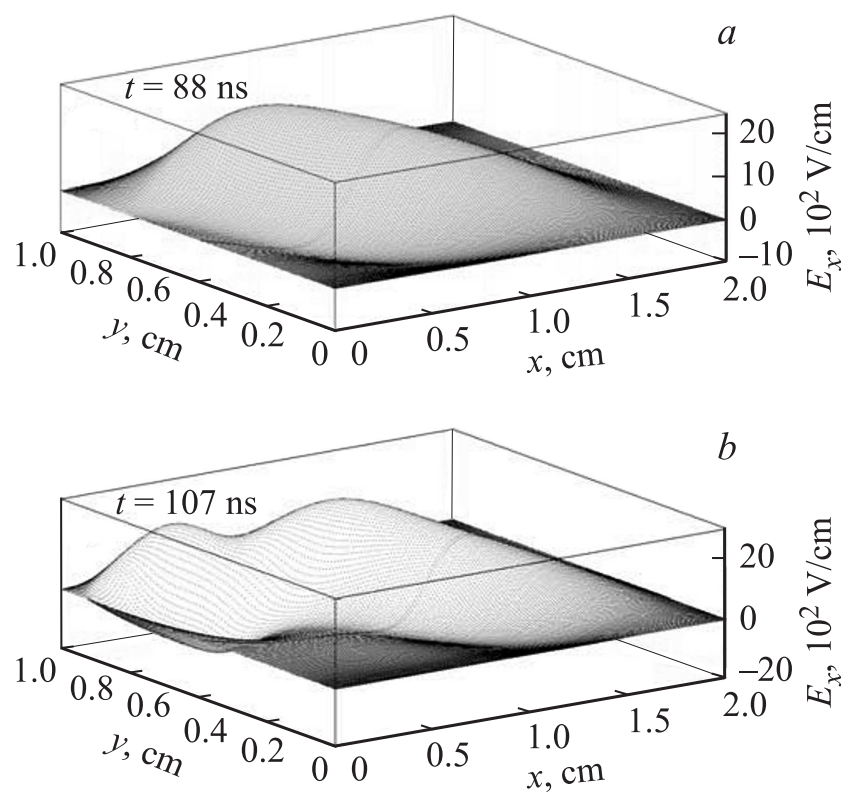

Рис. 3. Поперечная составляющая напряженности поля $E_{x}$ в межэлектродном промежутке: $U_{0}=7 \mathrm{kV}, p=760$ Torr, $d=1 \mathrm{~cm}$.

катоду напряженность поля на фронте ионизации также увеличивается, соответственно растет интенсивность ионизационных процессов. При уменьшении концентрации электронов предыонизации увеличиваются время формирования волны ионизации и соответственно время формирования разряда.

В сформировавшемся плазменном столбе концентрация заряженных частиц на оси разряда выше и уменьшается к периферии разряда. На интегральных картинах формирования разряда, полученных экспериментально, также можно заметить, что интенсивность излучения на оси разрядного промежутка выше и уменьшается от оси к периферии. Также следует отметить, что ток разряда на стадии формирования меньше, чем в случае однородной предыонизации, а концентрация электронов на оси разряда выше.

Письма в ЖТФ, 2017, том 43, вып. 18 
Таким образом, как показывают результаты экспериментальных исследований, в зависимости от внешних условий возможны различные режимы формирования и горения разряда, а именно однородный объемный разряд, объемный разряд с катодными пятнами, контрагированный разряд, сильноточный диффузный режим. Формирование разряда происходит в процессе развития катодонаправленной волны ионизации, что подтверждается как результатами численного моделирования, так и результатами экспериментальных исследований пространственновременно́й динамики развития начальных стадий разряда. Поперечная неоднородность предыонизации газа формирует неоднородный фронт волны ионизации, развивающейся из центральной зоны промежутка.

Выполненный в работе комплекс исследований указывает на сложность процессов на стадии формирования разряда, что определяет интерес дальнейшего изучения данной проблемы.

Работа выполнена при финансовой поддержке стипендией Президента РФ для молодых ученых и аспирантов № СП-3812.2016.1.

\section{Список литературы}

[1] Тарасенко В.Ф., Бакшт Е.Х., Бураченко А.Г. и др. // Письма в ЖТФ. 2010. T. 36. B. 8. C. $60-67$.

[2] Naidis G.V., Walsh J.L. // J. Phys. D: Appl. Phys. 2013. V. 46. N 9. P. 095203.

[3] Тарасенко В.Ф., Яковенко С.И. // УФН. 2004. Т. 174. № 9. С. 953-971.

[4] Бабич Л.П., Бочков Е.И., Куцык И.М. // Письма в ЖЭТФ. 2014. Т. 99. В. 7. C. $452-456$.

[5] Осипов В.В. // УФН. 2000. Т. 170. № 3. С. 225-245.

[6] Курбанисмаилов В.С., Омаров О.А., Рагимханов Г.Б. и др. // Физика плазмы. 2016. Т. 42. № 7. С. 680-692.

[7] Курбанисмаилов В.С., Омаров О.А. // Теплофизика высоких температур. 1995. T. 33. № 3. С. 346-350.

[8] Курбанисмаилов В.С., Омаров О.А., Хачалов М.Б. // Измерительная техника. 1989. № 3. С. 30-31.

[9] Kurbanismailov V.S., Omarov O.A., Ragimhanov G.B., Aliverdiev A.A. // Acta Polytechnica. 2015. V. 55. N 4. P. 237-241.

[10] Омаров О.А., Курбанисмаилов В.С., Арсланбеков М.А. и др. // Физика плазмы. 2012. Т. 38. № 1. С. 26-33.

[11] Курбанисмаилов В.С., Омаров О.А., Рагимханов Г.Б. // Успехи прикладной физики. 2015. Т. 3. № 2. С. 154-161.

Письма в ЖТФ, 2017, том 43, вып. 18 
[12] Терешонок Д.В. // Письма в ЖТФ. 2014. Т. 40. В. 3. С. 83-89.

[13] Райзер Ю.П. Физика газового разряда. Долгопрудный: Интеллект, 2009. $736 \mathrm{c.}$

[14] Суржиков C.T. Физическая механика газовых разрядов. М.: Изд-во МГТУ им. Н.Э. Баумана, 2006. 640 с.

[15] Soloviev V.R., Krivtsov V.M. // J. Phys. D: Appl. Phys. 2009. V. 42. N 12. P. 125208.

6 Письма в ЖТФ, 2017, том 43, вып. 18 